\title{
A Feasibility Pilot Study to Assess the Efficacy of NavX Guided Detec- tion of Pulmonary Vein Electrograms during Atrial Fibrillation Abla- tion [NavX Guided Detection of Pulmonary Vein Electrograms]
}

\author{
Yaariv Khaykin*, Pouria Alipour, Meysam Pirbaglou, Paul Ritvo, Zahra Azizi, Zaev Wulffhart, Bonnie \\ Whaley, David Giewercer, Kellie Winger and Atul Verma
}

Southlake Regional Heart Centre, Canada

*Corresponding author: Yaariv Khaykin, MD, FRCPC, FACC, FHRS, Southlake Regional Heart Centre, $206-581$ Davis Drive, Newmarket, Ontario, L3Y-2P6, Canada, Tel: 905-953-7917, Fax: 905-953-0046, E-mail: y.khaykin@utoronto.ca

\begin{abstract}
Background: Pulmonary Vein Antrum Isolation (PVAI) targeting $\mathrm{PV}$ triggers is an established treatment for paroxysmal Atrial Fibrillation (AF). Ablation lesions are typically delivered around the entire circumference of each individual PV. This study tested a strategy where electrical inputs to the PVs were mapped using the NavX system and selectively targeted before ablation. The objectives of the study were to evaluate: 1) Likelihood of PV isolation using this strategy, 2) Correlation between manually tagged PV potentials and Complex Fractionated Electrograms identified using the NavX CFE Mean Map in sinus rhythm and during coronary sinus pacing, 3) Long term success of this strategy.
\end{abstract}

Methods: Ablation was initially guided by identification of $\mathrm{PV}$ antral regions activating early in sinus rhythm and during Coronary Sinus (CS) pacing, and exhibiting local fractionated electrograms, mapped using the NavX system, and a circular mapping catheter. If $\mathrm{PV}$ isolation could not be established using this approach, it was completed using the conventional ablation approach. For analysis each vein was divided into quadrants from 12 o'clock looking from the Left Atrium (LA) into the vein. Manually identified fractionated potentials were compared with the NavX Complex Fractionated Electrogram (CFE) Mean map acquired in sinus rhythm and during coronary sinus pacing. CFE Mean Map parameters were set to a Width of 20 $\mathrm{ms}$, Refractory of $30 \mathrm{~ms}$, and P-P sensitivity of $0.05 \mathrm{mV}$. Manually tagged $\mathrm{PV}$ potentials were used as the gold standard. AF recurrences were assessed using ambulatory monitoring at 1 , $3,6 \& 12$ months post ablation.

Results: Twenty consecutive patients with paroxysmal AF (age $59 \pm 10 \mathrm{y}, 60 \%$ male, LA $40 \pm 6 \mathrm{~mm}$ ) were enrolled in the study. Distribution of CFE closely paralleled activation. In addition, CFE were identified on the septum in sinus rhythm in $60 \%$ of the patients and on the posterior wall during CS pacing in $35 \%$ of the patients. The sensitivity-specificity of the auto-identified CFE that predicted manually tagged fractionated potentials were $60 \%-68 \%$ in Sinus Rhythm (SR), and $80 \%-70 \%$ during CS pacing. Mapping and ablation fluoroscopy time was $30 \pm 12$ min with Radio Frequency (RF) time of $27 \pm 10 \mathrm{~min}$. Completion of PVAI required an additional $22 \pm 16 \mathrm{~min}$ of fluoroscopy and $31 \pm 28 \mathrm{~min}$ of RF energy delivery. Total procedure time was $209 \pm 36$ min. Only $13(4 \%)$ of all PV quadrants in $n=5(25 \%)$ patients did not have to be ablated to achieve PVAI. Three patients $(15 \%)$ had AF recurrences between the first 3 months and 12 months following ablation. Two of these patients cumulatively had 7 of the 13 non-ablated quadrants in the study.

Conclusions: While mapping earliest PV activation during sinus rhythm and CS stimulation is feasible, ablation guided by this approach may avoid unnecessary RF energy delivery in only a small proportion of the PV antral segments, potentially leading to higher ablation failure rates. NavX CFE mean algorithm accurately identifies areas of fractionation at PV antra during sinus rhythm and distal CS stimulation corresponding to PV potentials.

\section{Keywords}

Atrial fibrillation, Catheter ablation, Pulmonary vein antrum isolation
Abbreviations
ACT: Activated Clotting Time; AF: Atrial Fibrillation; AAD: Anti-Arrhythmic Drug; ICE: Intracardiac ECHO; LA: Left Atri- um; PV: Pulmonary Vein; PVAI: Pulmonary Vein Antrum Iso- lation; QoL: Quality of Life; TEE: Transesophageal ECHO; OAC: Oral Anti Coagulation

Citation: Khaykin Y, Alipour P, Pirbaglou M, Ritvo P, Azizi Z, et al. (2017) A Feasibility Pilot Study to Assess the Efficacy of NavX Guided Detection of Pulmonary Vein Electrograms during Atrial Fibrillation Ablation [NavX Guided Detection of Pulmonary Vein Electrograms]. Int J Clin Cardiol 4:102. doi.org/10.23937/23782951/1410102

Received: April 06, 2017; Accepted: September 23, 2017; Published: September 25, 2017

Copyright: (C 2017 Khaykin Y. This is an open-access article distributed under the terms of the Creative Commons Attribution License, which permits unrestricted use, distribution, and reproduction in any medium, provided the original author and source are credited. 


\section{Introduction}

Atrial Fibrillation (AF) is the most common cardiac arrhythmia [1]. It increases mortality risk, causes disability, reduces quality of life, and presents treatment challenges [2-4]. Sinus rhythm is typically preferred in clinical setting as opposed to $\mathrm{AF}$, in order to control patients' symptoms.

Established evidence implicates triggered activity in the Pulmonary Veins (PV), in initiating and maintaining $A F[5,6]$. Ablations of such foci of ectopic electrical activity have prevented AF recurrences and in some cases terminated chronic AF, altogether [7-11].

Early ablation strategies included direct focal mapping and elimination of pulmonary vein triggers in a time consuming process that resulted in low efficacy and in significant risks for pulmonary vein stenosis. Main stream approaches to ablation in patients with AF include electroanatomically guided pulmonary vein ablation $[9,11]$ and circumferential pulmonary vein (antrum) isolation using a circular mapping catheter [12], intracardiac echocardiography, and/or other non-fluoroscopic modalities as well as cryoballoon ablation. While these strategies achieve success in $60 \%-90 \%$ of patients, at low risk of hemorrhagic and embolic complications, and pulmonary vein stenosis following the procedure [12-14], most investigators feel that pulmonary vein isolation is an important endpoint of ablation that optimizes procedural success. Strategies aiming to isolate the pulmonary veins generally involve the need for double transseptal access in order to instrument the Left Atrium (LA) with a circular mapping catheter, as well as an ablation catheter. This may increase the risks associated with transseptal puncture and prolong fluoroscopy time during procedures. There has been no prospective testing of a strategy where electrical inputs to the pulmonary veins are mapped, with areas responsible for conduction into of the pulmonary vein antrum identified (on a 3D representation of the $L A$ ) and subsequently ablated using a single transseptal access.

NavX electroanatomical mapping system (St Jude Medical, Minneapolis, MN, USA), useful in the strategy described above, can be implemented to map conduction into the veins and help limit ablation lesions to solely the vein regions that propagate electrical impulses between the body of the left atrium and the PV antrum. It can also identify fractionated atrial electrograms using a CFE Mean Map software, which averages the timing intervals between multiple electrogram components over a user-defined time interval.

The objective of this feasibility pilot study was to test the strategy where electrical inputs to the PVs, defined by earliest PV activation in sinus rhythm and during distal Coronary Sinus (CS) stimulation, and/or by local fractionation, are selectively targeted to achieve PV antral isolation. A secondary objective was sensitivity-specific- ity analysis of the predictive ability of the CFE mean map to identify sites exhibiting local fractionation tagged by the operator, as well as fractionated areas identified using the NavX CFE algorithm.

\section{Methods}

Twenty consecutive patients with paroxysmal AF meeting criteria for ablation were prospectively enrolled into the study after signing consent. All patients completed 1-year follow-up. The study was approved by the institutional Ethics Review Board. The data was collected prospectively.

Patients received continuous oral anticoagulation with warfarin for at least three weeks prior to the experimental study. Warfarin was stopped 3 days prior to the procedure and the patients received low molecular weight heparin for bridging. They had a Transesophageal Echocardiogram (TEE) within 24 hours prior to the procedure to rule out left atrial thrombus or spontaneous echo-contrast.

All anti arrhythmic agents were discontinued at least 5 half-lives before the procedure or 6 months prior in the case of amiodarone.

During the procedure, a decapolar non-deflectable CS catheter was placed using the right internal jugular vein. An intracardiac echocardiography probe (ICE, Siemens AG Inc., Mountain View, CA, USA) was placed in the right atrium via the left femoral vein. Transseptal access to the left atrium was then established under fluoroscopic and ICE guidance. Patients received $5000 \mathrm{U}$ of unfractionated heparin IV with each of two transseptal punctures. Heparin infusion at $1000 \mathrm{U} /$ hour was initiated with the first puncture. Activated Clotting Time (ACT) was maintained in the range of 350-400 seconds.

Transseptal access was used to advance a decapolar circular mapping catheter (Lasso, Biosense Webster, Diamond Bar, CA, USA) and an irrigated tip ablation catheter (Celcius Thermocool, Biosense Webster, Diamond Bar, CA, USA) into the left atrium via braided SL-1 introducers (St Jude Medical, Minneapolis, MN, USA).

Once in the left atrium, 3D electroanatomic maps were constructed using NavX to display electrical activation in Sinus Rhythm (SR) and during distal CS stimulation. The NavX non fluoroscopic navigation system has been described elsewhere [15]. Briefly, the roving circular mapping catheter was placed $2-4 \mathrm{~cm}$ into each PV and slowly pulled back. Along pullback, multiple locations were recorded to tag the vein. The ostium was identified by fluoroscopic visualization of the catheter entering the cardiac silhouette with simultaneous impedance decrease and appearance of atrial potentials. Separate chamber geometries were collected for each pulmonary vein and for the left atrial appendage.

The NavX Local Activation Time map (LAT) was set up using a robust atrial reference signal from the proximal 
CS electrogram. The Roving Activation Interval (RAI) for the Complex Fractionated Electrogram (CFE) mean map was set to encompass only the atrial activation within the window of interest re: the reference signal, e.g. $-100 \mathrm{~ms}$ to $+200 \mathrm{~ms}$. Fractionated potentials in the roving catheter electrograms acquired during sinus rhythm and coronary sinus pacing could then be quantified using the CFE Mean map. CFE Mean Map parameters were set to a Width of $20 \mathrm{~ms}$, Refractory of $30 \mathrm{~ms}$, and P-P sensitivity set just above baseline noise, at $0.05 \mathrm{mV}$. Using this approach, LAT mapping resulted in a simultaneous acquisition of activation and fractionation maps

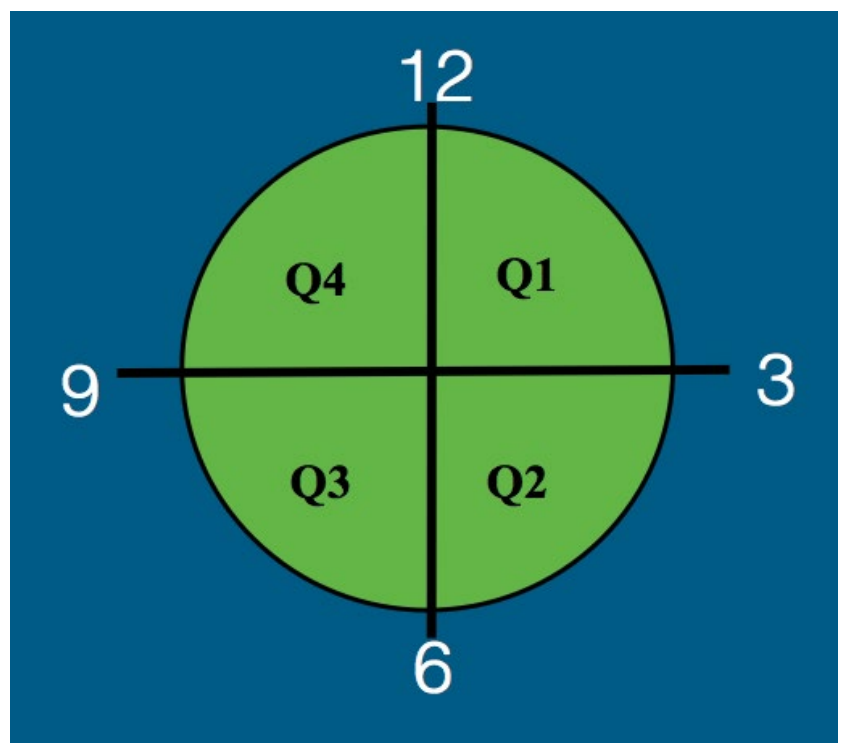

Figure 1: Representation of the pulmonary vein antrum for the analysis. Each vein was divided into quadrants clockwise from 12 o'clock, looking into the vein from the left atrium. in sinus rhythm and during distal CS stimulation. Each vein was divided into Quadrants for later analysis, looking from the LA into the vein and starting at 12 o'clock for Quadrant 1 (Figure 1).

Segments of earliest wave front propagation into the pulmonary vein antra were labeled on both maps, for sinus rhythm and for distal CS stimulation (Figure 2) using manual propagation of the activation front through the left atrium. Each acquired point was then examined manually by the operator for local electrogram fractionation defined as 3 or more high frequency local deflections (Figure 3). Fractionated sites were tagged for later comparison with the NavX CFE Mean Map. CFE were mapped during sinus rhythm and CS pacing. Only areas responsible for PV activation were ablated. No ablation was carried out beyond what was necessary to achieve PV isolation.

Ablation lesions were delivered using power of 3540 Watt for $\sim 20 \mathrm{sec}$ per lesion with the endpoint of disappearance of all local potentials in the areas of the pulmonary vein antrum defined by early activation in sinus rhythm and during CS pacing, or by the presence of fractionated potentials (Figure 4). After the initial set of lesions had been delivered, pulmonary vein antrum isolation was verified using the circular mapping catheter and additional ablation lesions were delivered as necessary to achieve PVAI (entrance block). Exit block was not routinely verified.

Sites tagged by the operator as exhibiting fractionation were then compared to the fractionated areas identified using the CFE Mean Map. Each patient received IV prota-

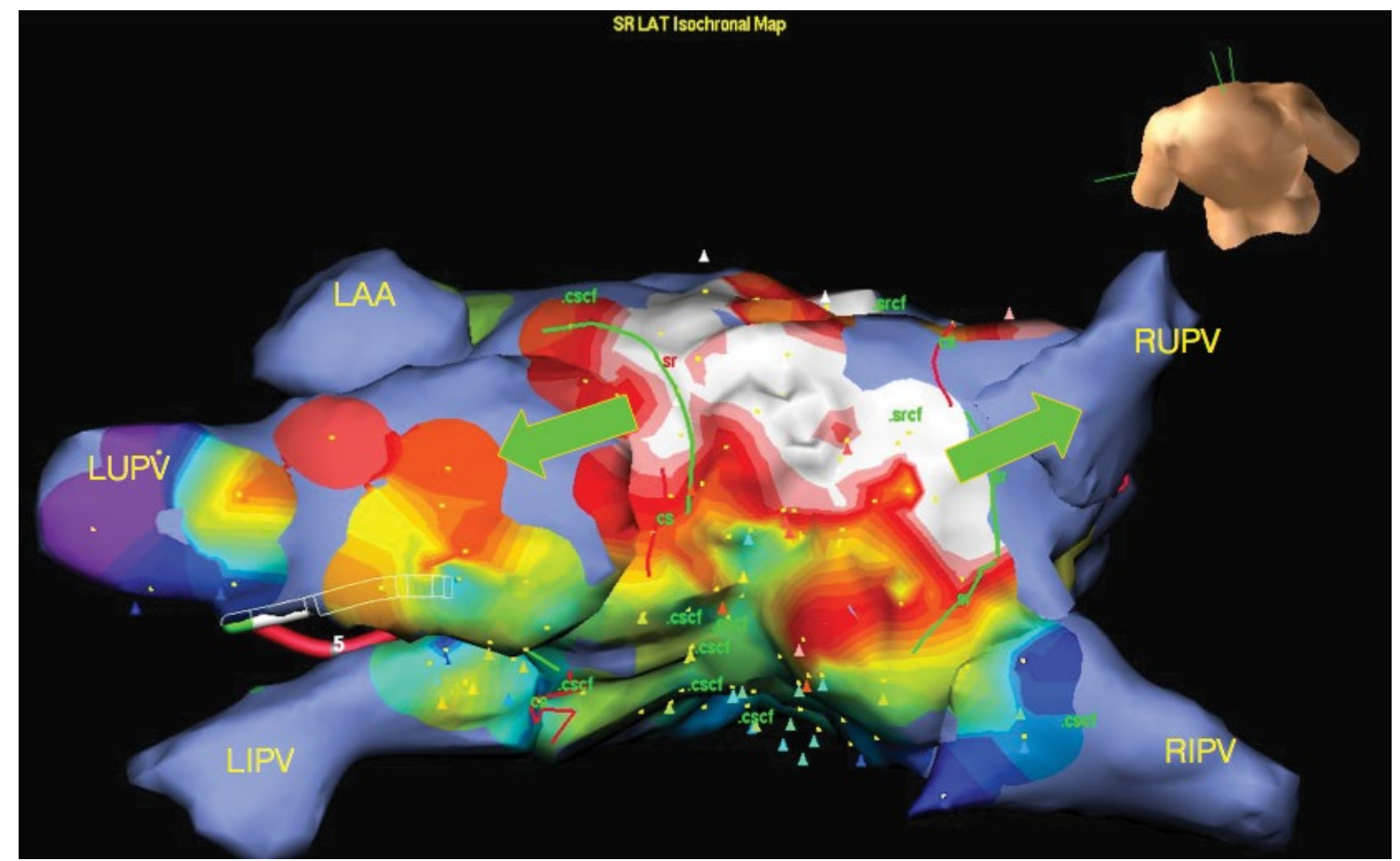

Figure 2: Wavefront propagation into pulmonary vein antra. Wavefront propagation was examined during manual propagation analysis of each map. Green lines represent early activating segments in sinus rhythm. Red lines represent early activating segments during distal CS stimulation. Green arrows illustrate the direction of wavefront propagation into the pulmonary veins. 

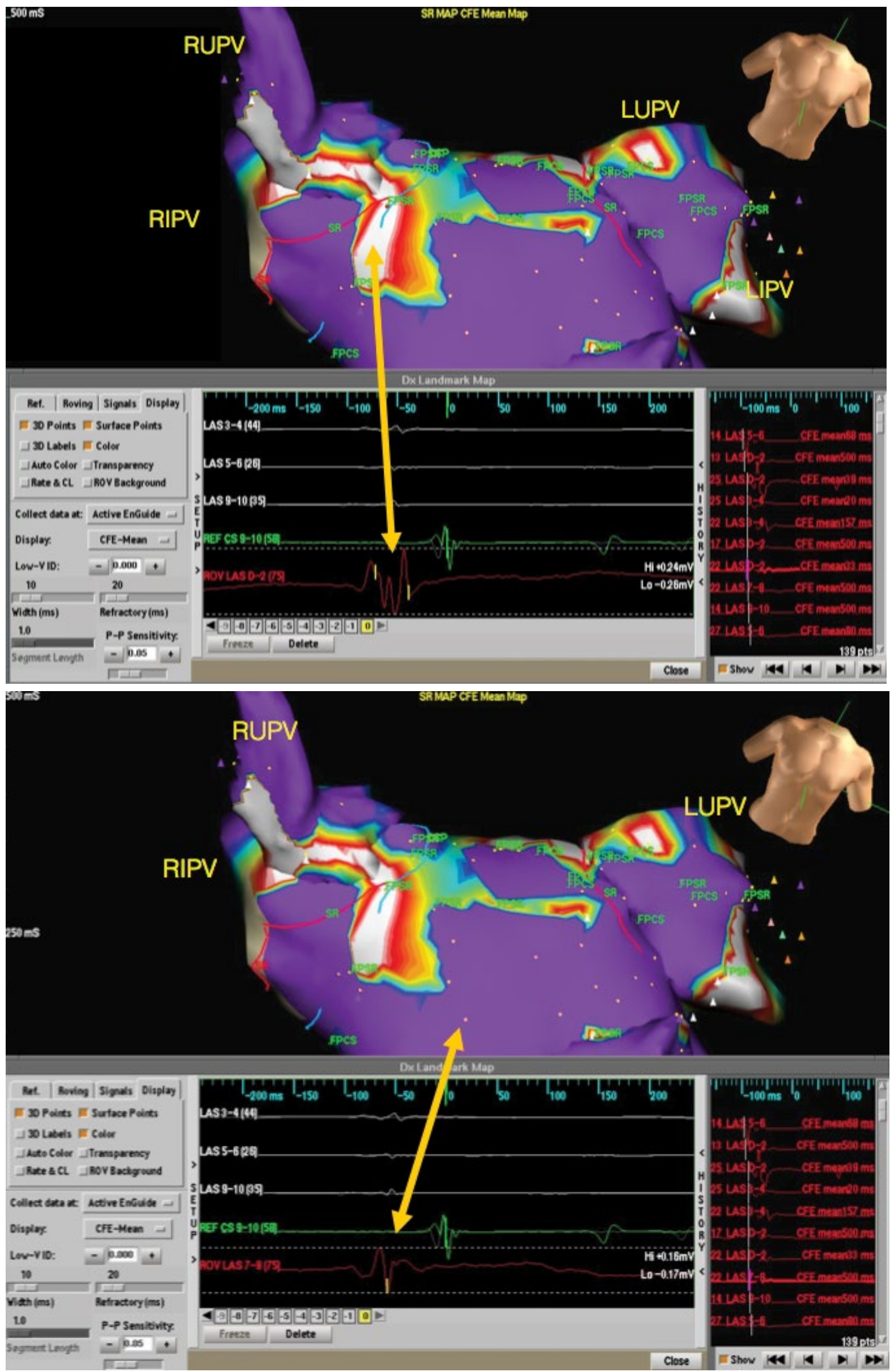

Figure 3: Correlation between manually tagged fractionated potentials, defined as 3 or more local deflections and the CFE mean map. Manually identified sites exhibiting local fractionation have been tagged and correspond to the white areas on the CFE mean map automatically identifying fractionation A) Manually identified fractionated area during sinus rhythm corresponds to the CFE Mean map and B) Manually identified non-fractionated area during sinus rhythm corresponds to the CFE Mean map. 


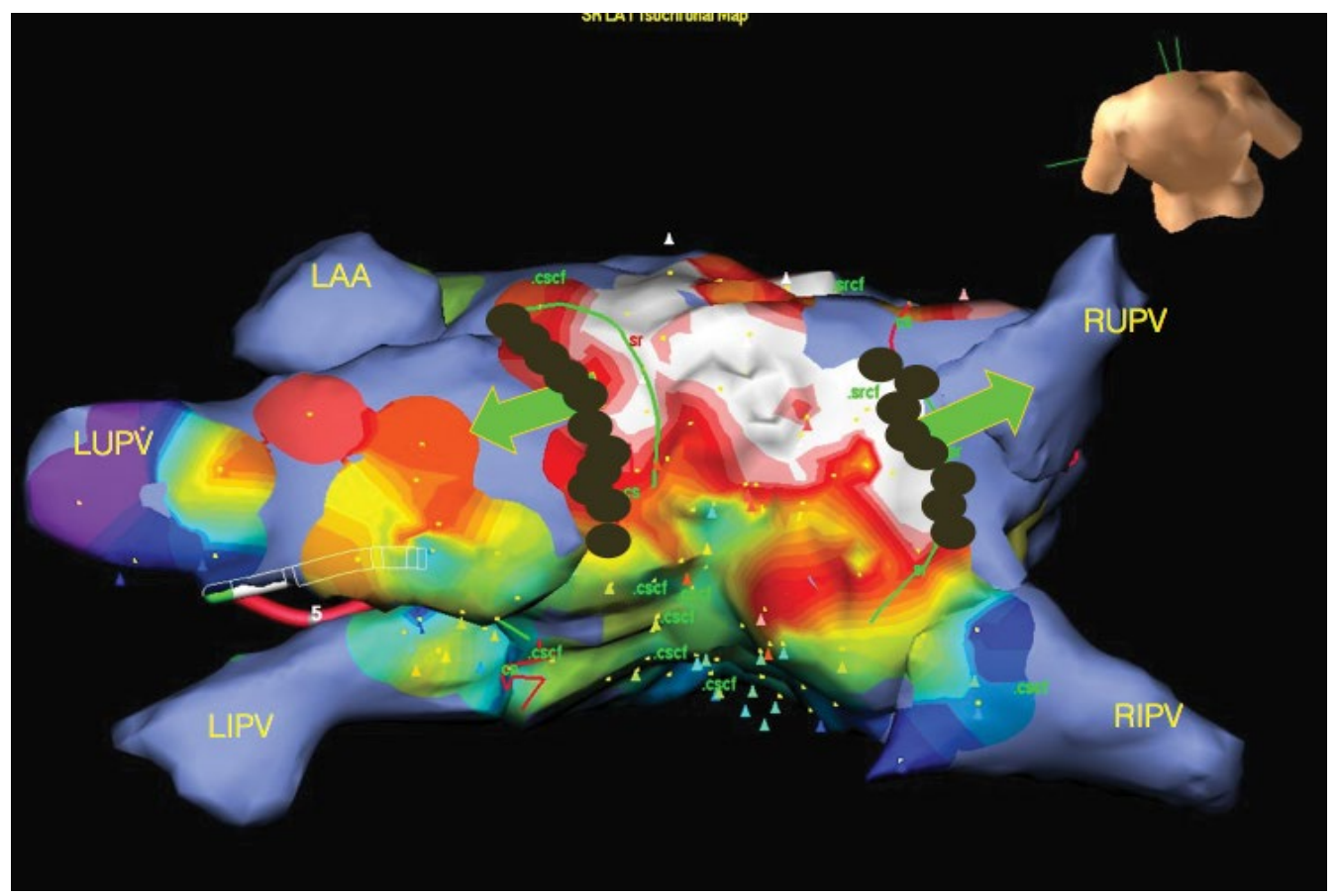

Figure 4: Part of the study lesion set targeting the segment of early wavefront propagation into the pulmonary veins in sinus rhythm. Green lines represent the early activating segments in sinus rhythm. Red lines represent early activating segments during distal CS stimulation. Green arrows illustrate the direction of wavefront propagation into the pulmonary veins.
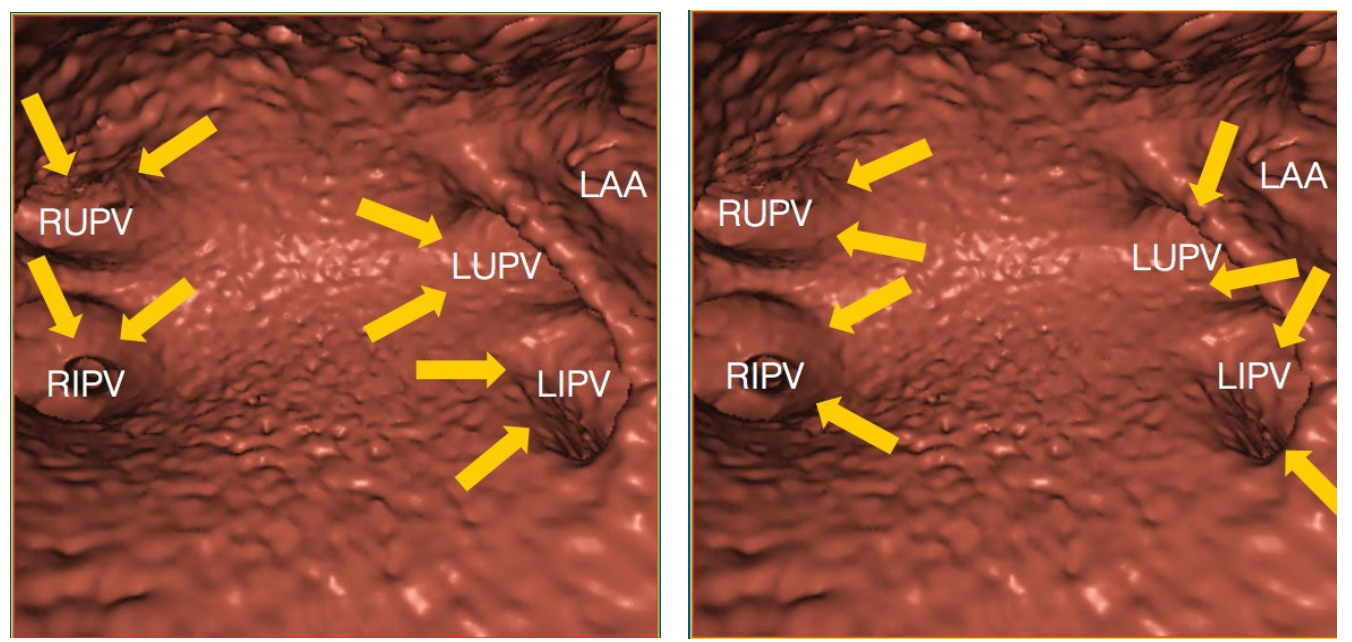

Figure 5: Pattern of activation of the pulmonary veins. Yellow arrows indicate direction of local activation at PV antra in sinus rhythm and during CS pacing A) During Sinus rhythm and B) During Distal CS stimulation.

LUPV: Left Upper PV; LIPV: Left Inferior PV; RUPV: Right Upper PV; RIPV: Right Inferior PV; LAA : Left Atrial Appendage.

mine upon withdrawal of the catheters into the right atrium to achieve ACT of $<200$ for sheath removal.

Patients were discharged home the day after ablation on oral anticoagulation with warfarin, without a bridging agent. Antiarrhythmic medications in patients taking these prior to ablation were resumed and continued for the first two months following ablation.

A spiral CT of the chest was used to rule out PV stenosis 3 months following ablation.

Recurrence of AF was assessed by a 24-hour Holter monitor assessment at 1, 3, 6 \& 12 months following ablation. Additional monitoring with a loop event recorder occurred in the presence of symptoms. Any episode of
AF $>30$ second's duration was defined as a recurrence. All data for this study was captured and analyzed prospectively from consecutive patients undergoing pulmonary vein isolation procedure at our institution.

\section{Results}

Baseline characteristics of the patients in the study are illustrated in Table 1. These are representative of the population of patients with paroxysmal AF ablated at our center [16].

\section{Fractionation and activation during mapping}

Left pulmonary veins activated preferentially along the posterior wall in sinus rhythm (Quadrants 3 and 4) 
and the ridge with LAA (Quadrants 1 and 2) during distal CS stimulation (Figure 5).

Right pulmonary veins were activated preferentially along the roof (Quadrants 4 and 1) during sinus rhythm and along the posterior wall during distal CS stimulation (Quadrants 1 and 2) for all patients in the study (Figure 5).

Distribution of fractionation closely paralleled that of activation. In addition, fractionated potentials were identified on the septum in sinus rhythm in $60 \%$ of the patients and on the posterior wall during distal CS stimulation in $35 \%$ of the patients.

\section{Sensitivity and specificity of the CFE mean map}

Sensitivity-specificity of the CFE mean map predicting manually identified fractionated PV potentials were $60 \%-68 \%$ respectively in SR and $80 \%-70 \%$ during distal CS stimulation. These were $70 \%-70 \%$ when evaluating the ability of the CFE mean map to predict manually tagged fractionated potentials during sinus rhythm or coronary sinus pacing (Table 2 ).

\section{Ablation}

Procedural parameters are shown in Table 3. Fluoroscopy and procedure times during study mapping and ablation were similar to those required to complete PVAl after the study protocol. Total procedure time was $209 \pm 36 \mathrm{~min}$. Total procedure and fluoroscopy times were in line with previously reported values.

Only $13(4 \%)$ of all pulmonary vein Quadrants in 5

Table 1: Pre-procedural characteristics, Mean \pm SD or $n(\%)$.

\begin{tabular}{|l|l|}
\hline Pre-procedural characteristics & Mean \pm SD or $\mathbf{n}(\%)$ \\
\hline Age (years) & $59 \pm 10$ \\
\hline Male Gender & $12(60)$ \\
\hline BMI & $29 \pm 6$ \\
\hline AF duration (years) & $6 \pm 6$ \\
\hline Number of failed AAD & $1.8 \pm 1.1$ \\
\hline LA size (mm) & $40 \pm 6$ \\
\hline Diabetes & $4(20)$ \\
\hline Hypertension & $8(40)$ \\
\hline
\end{tabular}

Table 2: Sensitivity and Specificity of the CFE mean map to predict manually identified fractionation.

\begin{tabular}{|l|l|l|l|}
\hline Location & Sensitivity & Specificity & P-Value \\
\hline Sinus rhythm & $60 \%$ & $68 \%$ & $\mathrm{P}<0.05$ \\
\hline Distal CS stimulation & $80 \%$ & $70 \%$ & $\mathrm{P}<0.05$ \\
\hline Either & $70 \%$ & $70 \%$ & $\mathrm{P}<0.05$ \\
\hline
\end{tabular}

Table 3: Procedural characteristics, Mean \pm SD.

Procedural characteristics Mean + SD

Mapping and study ablation protocol:

Fluoroscopy time (minutes)

RF time (minutes)

$30 \pm 12$

$27 \pm 10$

Additional ablation to achieve pvai after completion of the study protocol

Fluoroscopy time (minutes) $\quad 22 \pm 16$

RF time (minutes) $31 \pm 28$

Total procedure time (minutes)
(25\%) patients did not have to be ablated to achieve PVAI. Three patients (15\%) had AF recurrences between the first 3 months and 12 months following ablation. Two of these patients cumulatively had 7 of the 13 non-ablated quadrants in the study.

\section{Discussion}

This study explored a novel approach of sequential rather than concurrent mapping and isolation of the pulmonary vein antra guided by the NavX non-fluoroscopic 3D electroanatomical mapping system. Unfortunately, ablation could be avoided in only a small number of pulmonary vein antral segments. Patients who demonstrated antral isolation without the need for further ablation, had a higher likelihood of arrhythmia recurrence. In the remainder of the study group, completion of PVAI involved ablation of all other antral segments.

These findings support the perspective that complete pulmonary vein antral isolation is of paramount importance to AF ablation success. In most pulmonary veins, activation can proceed into the vein across any of the segments, depending on the source of the atrial rhythm. The areas showing earliest pulmonary vein antral activation frequently demonstrate local fractionated potentials, consistent with the "pulmonary vein potentials" typically targeted for ablation. While we don't have sufficient proof from this study that additional ablation required to complete the PVAI lesion set added to the success of the ablation, this may be hypothesized as most recurrences occurred in patients with evidence of pulmonary vein antral isolation, despite having segments of the antrum that were not ablated. These findings suggest that circumferential pulmonary vein antral isolation is superior to segmental ablation [13], contradicting some published evidence that indicates ablation of the antra can be limited to a few segments without sacrificing the clinical success of the procedure [17].

Similar potentials are also seen outside of the pulmonary veins at the sites of earliest left atrial activation, such as the septum during sinus rhythm, or the posterior wall during stimulation of the distal coronary sinus. The importance of these sites, as substrates for atrial fibrillation, has been previously demonstrated [18]. As observed in the results of this study the CFE mean map algorithm correlated very well with the human manual operator manual tagging fractionated signal corresponding to PV potentials which was used as the Gold Standard (Table 2). It is possible that the proprietary analytical system that presents "AF Nests" during sinus rhythm, that have been targeted for ablation [19], effectively identifies the same regions. In that case, such areas of interest could also be labeled with reasonable accuracy using the NavX CFE Mean algorithm. While there are a number of studies including STAR-AF II by Verma, et al. which demonstrated that ablation of CFEs mapped during AF have limited efficacy and in some cases, re- 
duces the success of AF ablation, in this study we did not intend to map or ablate areas tagged as exhibiting CFE beyond PV segments ablated to ensure isolation. Fractionated activity corresponding to PV potentials was mapped in sinus rhythm and during CS pacing rather than in AF. We then applied the NavX CFE Mean Map to the sinus rhythm and CS paced left atrial maps to find reasonable correlation between manually annotated PV-potential fractionation and fractionation as detected by the algorithm. Additionally, it is possible that the lesions delivered during the study were not transmural and complete. If this were the case, PVAl might have been achieved without additional ablation had complete lesions been delivered to the areas identified. For clinical purposes however, our technology remains limited and lesions were delivered using well-established RF energy settings.

\section{Limitations and Future Directives}

This was a prospective feasibility pilot study enrolling a small number of patients. Therefore there were a number of inevitable biases despite the small numbers we felt that further investigation would not be warranted based on our findings.

\section{Conclusions}

While mapping earliest PV activation during sinus rhythm and CS stimulation is feasible, ablation guided by this approach likely avoids unnecessary RF energy delivery in only a small proportion of PV antral segments. CFE Mean algorithm accurately identifies areas of fractionation at PV antra during sinus rhythm and distal CS stimulation. Full circumferential Pulmonary Vein Antrum ablation leading to isolation remains the preferred ablation strategy for AF refractory to medical therapy.

\section{Conflict of Interest Disclosures}

The Study was funded by a Research Grant from St Jude Medical. The Authors do not have any further individual disclosures with respect to this study.

\section{References}

1. Camm AJ, Obel OA (1996) Epidemiology and mechanism of atrial fibrillation and atrial flutter. Am J Cardiol 78: 3-11.

2. Benjamin EJ, Wolf PA, D'Agostino RB, Silbershatz H, Kannel WB, et al. (1998) Impact of atrial fibrillation on the risk of death: The framingham heart study. Circulation 98: 946952.

3. Wolf PA, Mitchell JB, Baker CS, Kannel WB, D'Agostino RB (1998) Impact of atrial fibrillation on mortality, stroke, and medical costs. Arch Intern Med 158: 229-234.

4. Connolly SJ (1999) Preventing stroke in atrial fibrillation: why are so many eligible patients not receiving anticoagulant therapy? CMAJ 161: 533-534

5. Haissaguerre M, Jais $P$, Shah DC, Takahashi A, Hocini M, et al. (1998) Spontaneous initiation of atrial fibrillation by ectopic beats originating in the pulmonary veins. $\mathrm{N}$ Engl $\mathrm{J}$ Med 339: 659-666.
6. Chen SA, Hsieh MH, Tai CT, Tsai CF, Prakash VS, et al. (1999) Initiation of atrial fibrillation by ectopic beats originating from the pulmonary veins: Electrophysiological characteristics, pharmacological responses, and effects of radiofrequency ablation. Circulation 100: 1879-1886.

7. Haissaguerre M, Shah DC, Jais P, Hocini M, Yamane T, et al. (2000) Electrophysiological breakthroughs from the left atrium to the pulmonary veins. Circulation 102: 2463-2465.

8. Natale A, Pisano E, Shewchik J, Bash D, Fanelli R, et al. (2000) First human experience with pulmonary vein isolation using a through- the-balloon circumferential ultrasound ablation system for recurrent atrial fibrillation. Circulation 102: 1879-1882.

9. Pappone C, Oreto G, Lamberti F, Vicedomini G, Loricchio ML, et al. (1999) Catheter ablation of paroxysmal atrial fibrillation using a 3d mapping system. Circulation 100: 1203-1208.

10. Pappone C, Oreto G, Rosanio S, Vicedomini G, Tocchi M, et al. (2001) Atrial electroanatomic remodeling after circumferential radiofrequency pulmonary vein ablation: Efficacy of an anatomic approach in a large cohort of patients with atrial fibrillation. Circulation 104: 2539-2544.

11. Pappone C, Rosanio S, Oreto G, Tocchi M, Gugliotta F, et al. (2000) Circumferential radiofrequency ablation of pulmonary vein ostia: A new anatomic approach for curing atrial fibrillation. Circulation 102: 2619-2628.

12. Marrouche NF, Dresing T, Cole C, Bash D, Saad E, et al. (2002) Circular mapping and ablation of the pulmonary veins for treatment of atrial fibrillation. Impact of different catheter technologies. J Am Coll Cardiol 40: 464-474.

13. Oral H, Scharf C, Chugh A, Hall B, Cheung P, et al. (2003) Catheter ablation for paroxysmal atrial fibrillation: Segmental pulmonary vein ostial ablation versus left atrial ablation. Circulation 108: 2355-2360.

14. Pappone C, Santinelli V (2001) Pulmonary vein isolation by circumferential radiofrequency lesions in atrial fibrillation from substrate to clinical outcome. Ann Ist Super Sanita 37: 401407.

15. Khaykin Y, Oosthuizen R, Zarnett L, Wulffhart ZA, Whaley $B$, et al. (2011) Carto-guided vs. Navx-guided pulmonary vein antrum isolation and pulmonary vein antrum isolation performed without 3-d mapping: Effect of the 3-d mapping system on procedure duration and fluoroscopy time. J Interv Card Electrophysiol 30: 233-240.

16. Khaykin Y, Oosthuizen R, Zarnett L, Essebag V, Parkash $\mathrm{R}$, et al. (2011) Clinical predictors of arrhythmia recurrences following pulmonary vein antrum isolation for atrial fibrillation: Predicting arrhythmia recurrence post-PVAI. J Cardiovasc Electrophysiol 22: 1206-1214.

17. Sy RW, Gula LJ, Leong-Sit P, Klein GJ, Yee R, et al. (2011) Complete antral encirclement is not required for pulmonary vein isolation. Heart Rhythm 8: 16-22.

18. Verma A, Patel D, Famy T, Martin DO, Burkhardt JD, et al. (2007) Efficacy of adjuvant anterior left atrial ablation during intracardiac echocardiography-guided pulmonary vein antrum isolation for atrial fibrillation. $\mathrm{J}$ Cardiovasc Electrophysiol 18: 151-156.

19. Bartalini S, Borri S, Galli I, Giusfredi G, Mazzotti D, et al. (2011) Measuring frequency noise and intrinsic linewidth of a room-temperature DFB quantum cascade laser. Opt Express 19: 17996-18003. 